\begin{tabular}{|c|c|c|c|}
\hline $\begin{array}{l}\text { 1. Publication No } \\
\text { INPE-4393-PRE } / 1213\end{array}$ & 2. Version & $\begin{array}{l}\text { 3. Date } \\
\text { Sept. } 1987\end{array}$ & \multirow{2}{*}{$\begin{array}{l}\text { 5. Distribution } \\
\square \text { Internal External } \\
\square \text { Restricted }\end{array}$} \\
\hline $\begin{array}{l}\text { 4. Origin } \\
L A P\end{array}$ & \multicolumn{2}{|c|}{$\begin{array}{l}\text { Program } \\
\text { PQUI }\end{array}$} & \\
\hline \multicolumn{4}{|c|}{$\begin{array}{l}\text { 6. Key words - selected by the author(s) } \\
\text { RAREFACTIVE SOLITONS } \\
\text { ION-ACOUSTIC SOLITONS } \\
\text { PLASMA WITH NEGATIVE IONS }\end{array}$} \\
\hline \multicolumn{4}{|c|}{ 7. U.D.C.: 533.9} \\
\hline \multirow{3}{*}{\multicolumn{3}{|c|}{$\begin{array}{l}\text { 8. Title } \\
\text { INPE-4393-PRE/1213 } \\
\text { EXPERIMENTS ON ION-ACOUSTIC RAREFACTIVE } \\
\text { SOLITONS IN A MULTI-COMPONENT PLASMA } \\
\text { WITH NEGATIVE IONS }\end{array}$}} & 10. No of pages: 13 \\
\hline & & & 11. Last page: 12 \\
\hline & & & 12. Revised by \\
\hline \multirow{2}{*}{\multicolumn{3}{|c|}{$\begin{array}{ll}\text { 9. Authorship } & \text { Y. Nakamura } \\
& \text { J.L. Ferreira } \\
\text { G.O. Ludwig }\end{array}$}} & $\begin{array}{l}\text { Autrow Aouls } \\
\text { Antonio Montes }\end{array}$ \\
\hline & & & $\begin{array}{l}\text { Manko Antonio Rawp } \\
\text { Dipector General }\end{array}$ \\
\hline \multicolumn{4}{|c|}{$\begin{array}{l}\text { Ion-acoustic solitons in a three-component plasma which } \\
\text { consists of electrons and positive and negative ions have been } \\
\text { investigated experimentaliy. When the concentration of negative ions } \\
\text { is smaller than a certain value, positive or compressive solitons are } \\
\text { observed. At the critical concentration, a broad pulse of small but } \\
\text { finite amplitude propagates without changing its shape. When the } \\
\text { concentration is larger than this value, negative or rarefactive } \\
\text { solitons are excited. The velocity and the width of these solitons are } \\
\text { measured and compared with predictions of the Korteweg-de Vries } \\
\text { equation which takes the negative ions and the ion temperature into } \\
\text { consideration. Head-on and over-taking collisions of the rarefactive } \\
\text { solitons have been observed to show that the solitons are not affected } \\
\text { by these collisions. }\end{array}$} \\
\hline \multicolumn{4}{|c|}{$\begin{array}{l}\text { 15. Remarks } \\
\text { (1985), 237-248. }\end{array}$} \\
\hline
\end{tabular}




\title{
Experiments on ion-acoustic rarefactive solitons in a multi-component plasma with negative ions
}

\author{
By Y. NAKAMURA, \\ Institute of Space and Astronautical Science, \\ Komaba, Megaro-ku,Tokyo 153, Japan

\section{J. L. FERREIRA AND G. O. LUDWIG} \\ Instituto de Pesquisas Espaciais, \\ Conselho Nacional de Desenvolvimento Cientifico e Tecnológico, \\ 12200 São José dos Campos, São Paulo, Brazil \\ (Received 3 November 1984 and in revised form 2 January 1985)
}

Ion-acoustic solitons in a three-component plasma which consists of electrons and positive and negative ions have been investigated experimentally. When the concentration of negative ions is smaller than a certain value, positive or compressive solitons are observed. At the critical concentration, a broad pulse of small but finite amplitude propagates without changing its shape. When the concentration is larger than this value, negative or rarefactive solitons are excited. The velocity and the width of these solitons are measured and compared with predictions of the Korteweg-de Vries equation which takes the negative ions and the ion temperature into consideration. Head-on and overtaking collisions of the rarefactive solitons have been observed to show that the solitons are not affected by these collisions.

\section{Introduction}

Ion-acoustic solitons have been frequently investigated experimentally over a period approaching a score of years. Experiments originally for planar and one-dimensional solitons have been extended to cylindrical and spherical cases and to oblique collisions of two propagating solitons. Various aspects of these soliton phenomena have been reviewed by several papers (Ichikawa \& Watanabe 1977; Ikezi 1978; Tran 1979; Nakamura 1982; Lonngren 1983). These solitons are compressions of plasma density, i.e. their electric potential is positive. A broad compressive perturbation of large amplitude breaks up into a number of solitons as it propagates, whereas a rarefactive perturbation generates a subsonic wave train (Okutsu \& Nakamura 1979). No rarefactive or negative soliton exists in a plasma composed of positive ions and electrons.

Das \& Tagare (1975) and Das (1977) have theoretically derived the Kortewegde Vries equation for nonlinear ion-acoustic waves in a plasma which contains contaminating negative ions from a fluid model of the plasma. They showed that rarefactive or negative solitons can propagate when the concentration of negative ions is larger than a certain critical value which depends on the mass 
ratio of the negative ions to positive ones and on the temperature ratio of ions to electrons. The rarefactive soliton has not been observed so far except as reported in Ludwig, Ferreira \& Nakamura (1984). It is the purpose of the present paper to report experimental results on the propagation of rarefactive solitons and to compare them with predictions of the Korteweg-de Vries (KdV) equation. The linear dispersion relation and the $K d V$ equation for ion-acoustic waves for the plasma with negative ions are introduced in $\$ 2$. In $\S 3$ the experimental procedure is described. The experimental results are stated and discussed in $\$ 4$. $\$ 5$ gives the conclusions.

\section{Theory}

The dispersion relation for ion-acoustic waves in a three-component plasma which has one species of contaminating negative ions is easily obtained from the fluid model and is given by

$$
1+\frac{1}{K^{2}}=\frac{1}{\Omega^{2}-3(1-r) K^{2} T}+\frac{r}{\mu \Omega^{2}-3(1-r) K^{2} T},
$$

where $K=k / k_{e}, \Omega=\omega / \omega_{p i}, T=T_{i} / T_{e}, \mu=M_{-} / M_{+}, k_{e}=\left(4 \pi n e^{2} / \kappa T_{e}\right)^{\frac{1}{2}}$ and $\omega_{p i}=\left(4 \pi n_{+} e^{2} / M_{+}\right)$. Furthermore, $M_{-}$and $M_{+}$are the mass of the negative and positive ions, respectively, $n$ and $n_{+}$are the density of the electrons and the positive ions, respectively, and $r=n_{-} / n_{+}$. Here charge neutrality, i.e. $n_{+}=n+n_{-}$, where $n_{-}$is the density of the negative ions, and equal temperatures for the positive and negative ions are assumed.

The phase velocity $\omega / k$ when $K \ll 1$ obtained from (1) has two values. When $\mu>3 T$, which applies in the present experimental conditions, one mode, called a fast mode, becomes the ion-acoustic wave of the positive ion when $r=0$ and the other is a virtual mode with $\omega / k=\left(3 \kappa T_{i} / M_{-}\right)^{\frac{1}{2}}$ at the limit $r=0$ (D'Angelo, Goeler \& Ohe 1966). The fast mode has been observed experimentally, and its ion-acoustic velocity $C_{s}($ i.e. $\omega / k$ when $K \ll 1$ ) was found to agree with the predietion of (1) (Wong, Mamas \& Arnush 1975; Ludwig et al. 1984).

The KdV equation for the three-component plasma, derived from the fluid model which takes finite and equal ion temperatures into consideration, is written as (Das 1977, 1979)

$$
\frac{\partial \psi}{\partial \tau}+\frac{A}{B} \frac{\psi \partial \psi}{\partial \xi}+\frac{1}{2 B} \frac{\partial^{3} \psi}{\partial \xi^{3}}=0
$$

where

$$
\begin{aligned}
& A=\frac{3}{2(1-r)}\left[\frac{S^{2}+T}{\left(S^{2}-3 T\right)^{3}}-\frac{r\left(\mu S^{2}+T\right)}{\left(\mu S^{2}-3 T\right)^{3}}\right]-\frac{1}{2}, \\
& B=\frac{S}{1-r}\left[\frac{1}{\left(S^{2}-3 T\right)^{2}}+\frac{\mu r}{\left(\mu S^{2}-3 T\right)^{2}}\right],
\end{aligned}
$$

$\psi$ is the perturbed electron density $\delta n$ normalized to $n, S=C_{s} /\left(\kappa T_{e} / M M_{+}\right)^{t}$, $\tau=k_{e} C_{s} t$ and $\xi=k_{e}\left(x-C_{s} t\right)$. In the previous paper (Ludwig et al. 1984), the ion temperature was assumed to be zero. 
Equation (2) has a soliton solution given by

$$
\psi=\psi_{0} \operatorname{sech}^{2}\left[k_{e}\left(\frac{A \psi_{0}}{6}\right)^{\frac{t}{t}}\left(x-C_{s} t-\frac{A \psi_{0} C_{s} t}{3 B S}\right)\right]
$$

where $\psi_{0}$ is the height or depth of solitons. The Mach number $M$ of solitons, i.e. the velocity divided by the ion-acoustic speed $C_{s}$, is

$$
M=1+A \psi_{0} / 3 B \dot{S},
$$

which indicates that the velocity increment from $C_{b}$ is proportional to the amplitude. The width of solitons normalized to the electron Debye length $\lambda_{e}\left(=1 / k_{e}\right)$ satisfies the relation

$$
\left(D / \lambda_{e}\right)^{2} \psi_{0}=6 / A .
$$

Equation (4) shows that the square of the width times the amplitude is constant. The above two relations are definite characteristics of KdV solitons, which are often used to identify solitary waves observed experimentally.

The coefficient of the nonlinear term in the KdV equation (2) vanishes at a certain value of $r$, which is determined from $A=0$, for fixed $\mu$ and $T$. The value of $B$ is positive in the whole range of $r$. Therefore, when $r$ is smaller than the critical value, compressive solitons can propagate since the coefficient is positive. When $r$ is equal to the critical value $r_{c}$ which is 0.102 when $\mu=0.476$ for $\mathrm{Ar}^{+}$and $\mathrm{F}^{-}$ions and $T=0$, the nonlinear term vanishes and the $\mathrm{KdV}$ equation becomes linear. When $r>r_{c}$, as the coefficient is negative, rarefactive solitons are expected to be observed.

The existence of negative solitons is interpreted as follows. A relation which indicates the phase between the perturbed density $\delta n_{f}$ and the wave potential $\phi$ is easily obtained from the equation of motion and the continuity equation used for the derivation of (1), and is given by

$$
\frac{\partial n_{j}}{n_{j}}\left(v_{p}^{2}-\frac{\gamma_{j} \kappa T_{j}}{M_{j}}\right)=\frac{q_{j} \phi}{M_{j}}
$$

Here the subscript $j$ implies each species of the plasma component, $q_{j}$ is the electric charge, $M_{j}$ is the mass, $n_{j}$ is the unperturbed density and $v_{p}$ is the phase velocity. The ratio of specific heats $\gamma_{j}$ is 1 for electrons and 3 for both positive and negative ions. For the present wave, since

$$
v_{p}^{2} \ll \kappa T_{\epsilon} / M_{\epsilon} \text { and } v_{p}^{2} \gg 3 \kappa T_{-} / M_{-}>3 \kappa T_{+} / M_{+},
$$

$\delta n_{+}$and $\delta n_{\epsilon}$ are in phase with $\phi$, and $\delta n_{-}$is out of phase with $\phi$. Therefore, a rarefactive wave pulse (rarefaction of positive ions and electrons) which has a negative potential is related to a compression of negative ions. The nonlinearity, i.e. the displacement of the negative ions in the pulse, steepens the wave shape at the leading edge when the negative ion density is larger than a critical value. The steepening is balanced by a negative dispersive effect to form a negative soliton. 


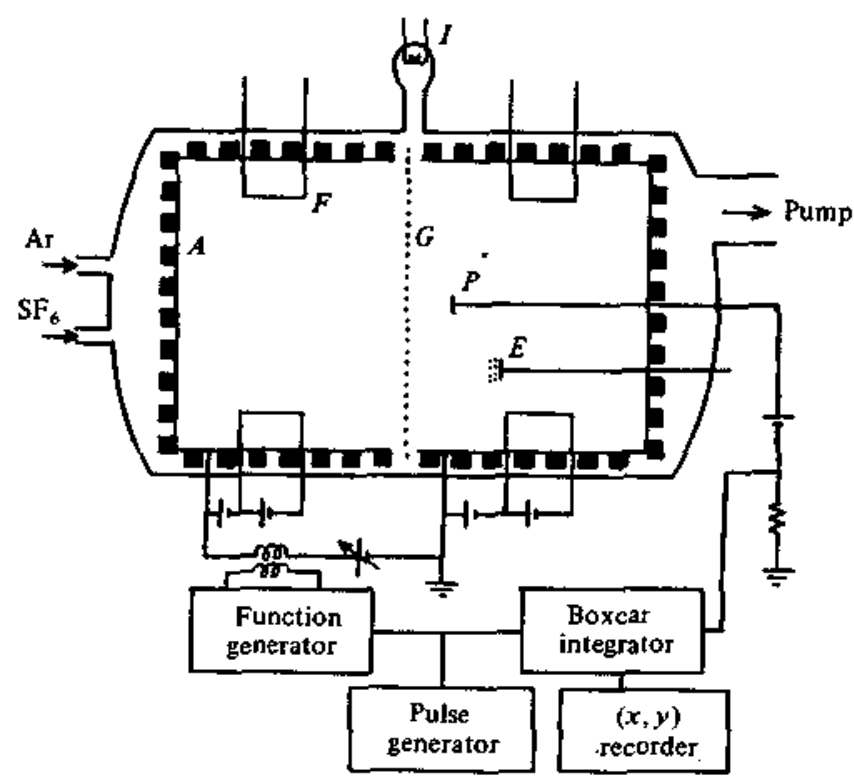

Figure 1. Schematic diagram of the experimental apparatus. $A$ : magnets enclosed in thin aluminium pipes which are used as the anode. $E$ : retarding energy analyser. $F$ : filaments. $G$ : floating grid. $I$ : ionization gauge. $P$ : plane Langmuir probe.

\section{Experimental procedure}

The experiment was carried out in a multi-dipole double-plasma machine shown schematically in figure 1. The inner diameter (plasma diameter) of the device is $40 \mathrm{~cm}$ and its total length is $90 \mathrm{~cm}$. The chamber is divided into a source and a target section with a floating grid. The grid consists of a mesh (20 lines per inch) made of $0.1 \mathrm{~mm}$ molybdenum wires. The cathodes consist of $0.1 \mathrm{~mm}$ diameter tungsten filaments and are placed at $6 \mathrm{~cm}$ from the surface of the anodes. Each plasma section has fifteen filaments with a length of $5 \mathrm{~cm}$. The chamber is evacuated down to $1 \times 10^{-6}$ torr by a turbo-molecular pump. Argon and sulphur hexafluoride are introduced into the chamber under continuous pumping. The maximum partial pressures of argon and sulphur hexafluoride are $1.5 \times 10^{-4}$ torr and $4 \times 10^{-5}$ torr, respectively. The discharge voltage is $50 \mathrm{~V}$ and the discharge current in each section is $70-150 \mathrm{~mA}$. Plasma parameters are measured by a plane Langmuir probe with a diameter of $5 \mathrm{~mm}$ and a retarding ion energy analyser. The back of the probe is covered with a ceramic insulator. The electron density $n=10^{8}-10^{9} \mathrm{~cm}^{-3}$, the electron temperature $T_{e}=1 \cdot 5-2 \cdot 3 \mathrm{eV}$ and the electron to positive ion temperature ratio $T_{e} / T_{i}=10-20$.

Ion-acoustic perturbations are excited by either a positive or negative sinusoidal voltage pulse which is applied to the anode of the driver section. Signals are detected with the axially movable plane Langmuir probe. It is biased positively against the plasma potential to collect the electron saturation eurrent and is therefore sensitive to the perturbed electron density. The signals 


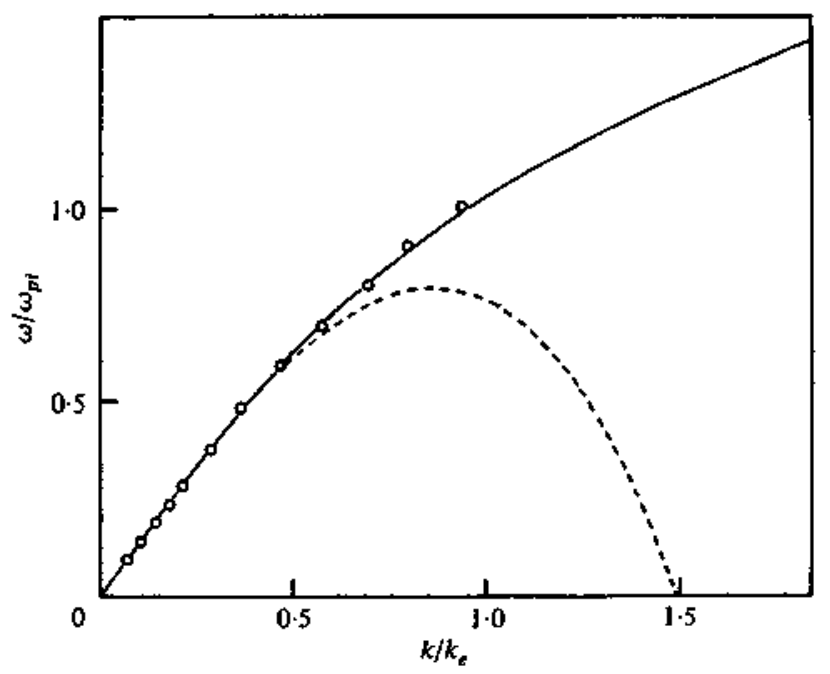

Figure 2. A measured dispersion relation. Argon pressure $=1.1 \times 10^{-4}$ torr. $S_{6}$ pressure $=1.5 \times 10^{-5}$ torr. - - estimated fast mode from (1) for $r=0.34$ and $T_{i} / T_{c}=0.07, \ldots$, linearized (2) for the same $r$ and $T_{i} / T_{\epsilon}$.

are fed into either an oscilloscope or a boxcar integrator whose output is recorded on an $(x, y)$ recorder versus position.

\section{Experimental results and discussion}

Bombardments of $\mathrm{SF}_{6}$ molecules by $50 \mathrm{eV}$ primary electrons lead mainly to the formation of negative $\mathbf{F}$ - ions due to dissociative attachment processes (Asundi \& Craggs 1964). Negative $\mathrm{SF}_{6}^{-}$and $\mathrm{SF}_{5}^{-}$ions are also formed by the capture of low-energy plasma electrons, and cross-sections indicate that for an electron temperature of $2 \mathrm{eV}$ the production rate of $\mathrm{SF}_{5}^{-}$ions is much larger than $\mathrm{SF}_{6}^{-}$ions. Furthermore, the ionization of $\mathrm{SF}_{6}$ molecules by primary electrons leads to the formation of positive ions such as $\mathrm{SF}_{5}^{+}, \mathrm{SF}_{3}^{+}, \mathrm{SF}_{4}^{+}$, ete. Therefore, the plasma consists of several species of positive and negative ions. However, it is easily seen from the dispersion relation of ion-acoustic waves in a plasma which includes multi-component of ions that the phase velocity of the fast mode depends strongly on light ion concentration, which has been confirmed by experiments (Tran \& Coquerand 1976; Nakamura, Nakamura \& Itoh 1976). The coefficient of the nonlinear term in (2) also depends mainly on light ion concentrations (Ludwig et al. 1980). Hence we assume that the plasma is composed of $\mathrm{Ar}^{+}$ions, $\mathrm{F}^{-}$ions and electrons and the contribution of other heavier positive and negative ions is neglected. The method of obtaining the concentration of $\mathrm{F}^{-}$-ions experimentally from a measured dispersion relation is described below.

An example of measured dispersion relations is shown in figure 2. The solid curve is obtained from (1) where $r$ and $T$ are chosen to give the best fit to the experimental result. The temperature and density of electrons used for the calculation have been measured by the Langmuir probe. As a result of this, the 


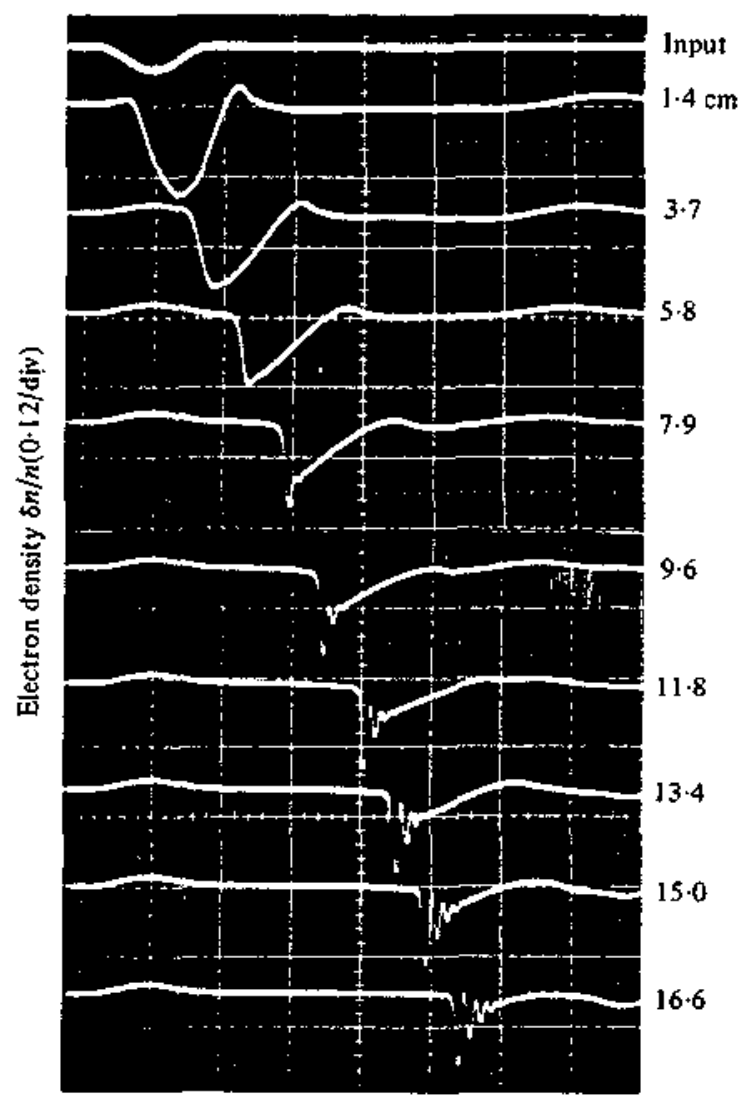

Time $(10 \mu \mathrm{s} / \mathrm{div})$

FIgURe 3. Electron density perturbation versus time with the distance from the grid as a parameter. $r \simeq 0.23$. The top trace is the applied signal.

$F^{-}$concentration $r$ is obtained. The dotted eurve is the linearized (2), which is calculated for the same parameters as the solid curve. Both curves agree when $k / k_{e} \leqslant 0 \cdot 5$, for the reason that the $\mathrm{KdV}$ equation is valid for the perturbation whose characteristic length is much longer than the Debye length.

An example of observed signals for a negative initial pulse whose width is much wider than $1 / k_{e}$ when $r>r_{c}$ is shown in figure 3 . When the sign of the signals is inverted, development of the pulse is similar to the case of a compressive pulse in a plasma without negative ions, which was observed and compared with numerical integrations of the $\mathrm{KdV}$ equation by Okutsu \& Nakamura (1979). The leading part of the pulse steepens since it propagates faster and since the effect of dispersion is much smaller than the nonlinearity (3.7 and $5.8 \mathrm{~cm}$ in figure 3 ;. The steepening caused by the nonlinearity makes the role of dispersion larger and the first soliton is created when the nonlinearity balances the dispersion $(7.9$ and $9.6 \mathrm{~cm})$. At the same time, the second soliton begins to emerge, and so on. The humps seen at the time of the applied pulse are directly coupled signals (Nakamura \& Nomura 1980). Under the same plasma condition, a positive pulse has been transmitted. As the pulse propagates, the 


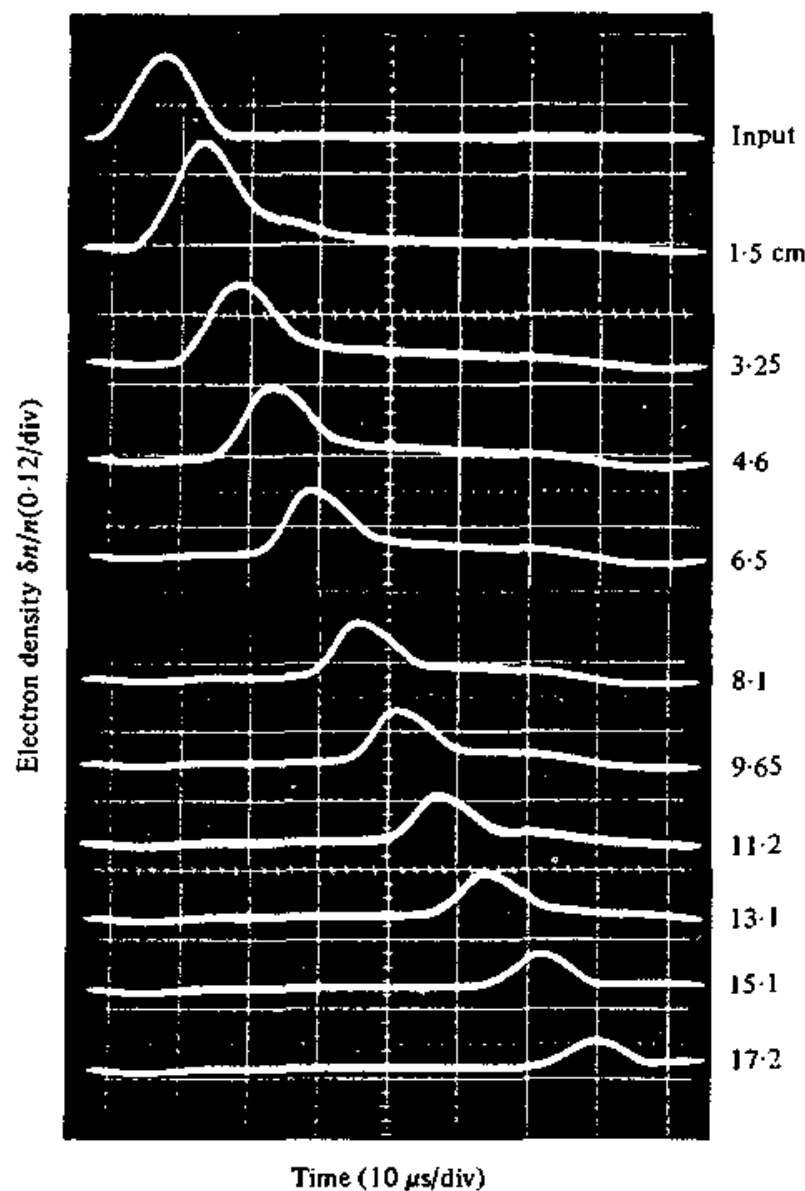

Froure 4. Wave response to a pulse excitation for several distances from the grid as a parameter. $r \simeq 0 \cdot 14$.

leading part becomes gentle and steepening occurs not at the rising (leading) part but at the falling part of the pulse. As a result of this, oscillations instead of solitons appear eventually. This phenomenon is the counterpart of the propagation of a rarefactive pulse in a plasma with no negative ions, which breaks up into oscillations (Okutsu \& Nakamura 1979). The result also suggests that the coefficient of the nonlinear term in the $K d V$ equation is negative in the present plasma condition $\left(r \simeq 0 \cdot 23>r_{c}\right)$.

Observation of the propagation of a positive pulse when $r \simeq r_{c}$ is shown in figure 4. Although the amplitude of the pulse is not small enough $(\delta n / n \simeq 0 \cdot 1)$ to be considered linear, it does not show steepening at the leading edge but propagates like a linear wave without change of its shape except by damping. This is because the nonlinear term of the KdV equation disappears. When the amplitude is increased further, a higher-order term of nonlinearity should be considered, which results in the modified $\mathrm{KdV}$ equation (Nakamura \& Tsukabayashi 1984). The measured critical concentration $r_{c}(=0 \cdot 14 \pm 0 \cdot 04)$ agrees with 


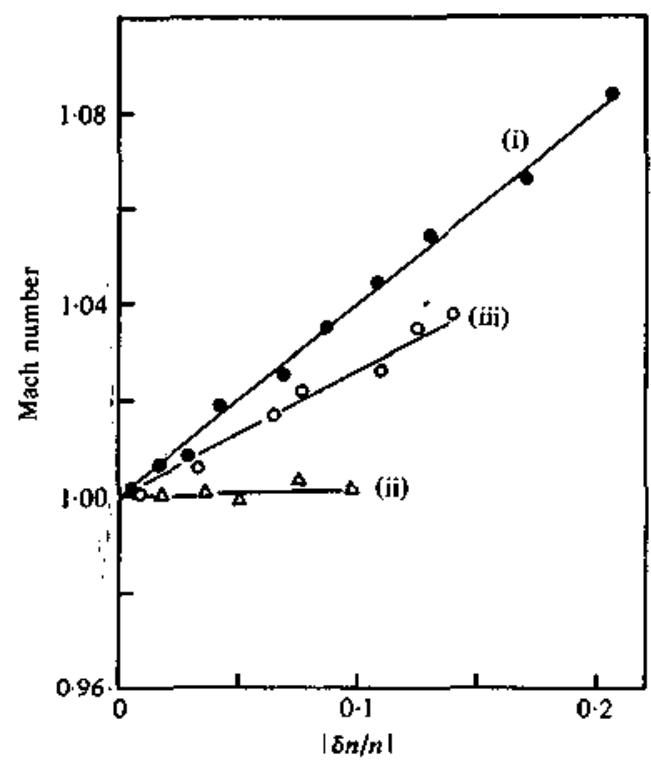

Figdre 5. Measured Mach numbers of (i) compressive solitons $(r=0)$, (ii) positive pulses $(r=0.13)$ and (iii) rarefactive solitons $(r=0.42)$ as a function of the normalized ampli. tude.

the prediction of (2), which confirms that the dominant species of ions are $\mathrm{Ar}^{+}$ and $\mathrm{F}^{-}$.

The measured velocities of compressive solitons when $r=0$, rarefactive solitons when $r=0.42$ and of the positive pulses when $r=0.13$ are plotted in figure 5 as a function of the normalized amplitude. The velocity is measured only for the first soliton shown in figure 3 since the adjoining second and the third solitons are not separated completely yet (Okutsu \& Nakamura 1979). The height or depth of solitons is varied by changing the excitation voltage applied to the anode of the source chamber. For both compressive and rarefactive solitons, the result indicates that $(M-1)$ is linearly proportional to the amplitude, which is consistent with (3) and which has already been measured for compressive solitons in plasmas without negative ions (Ikezi 1973; Watanabe 1975; Okutsu \& Nakamura 1979).

When $r=0.13$ which is a little smaller than the experimental $r_{c}(0 \cdot 14)$, the measured wave is not a soliton but a positive pulse. However, the velocity increment is also proportional to its amplitude as explained below. Since the width of the pulse is much wider than the Debye length, the dispersion term in (2) is much smaller than the nonlinear term within a distance of $15 \mathrm{~cm}$, where the velocity has been measured. As a result of this, (2) predicts that the velocity of the peak of the pulse in the frame of reference moving with the ion-acoustic speed is proportional to its height.

The gradients of lines like those in figures 5 , which are equal to $(M-1) /(\delta n / n)$, are measured and summarized in figure 6 . The open circle on the vertical axis is the case when $r=0$, i.e. the plasma includes no negative ions. The solid and 


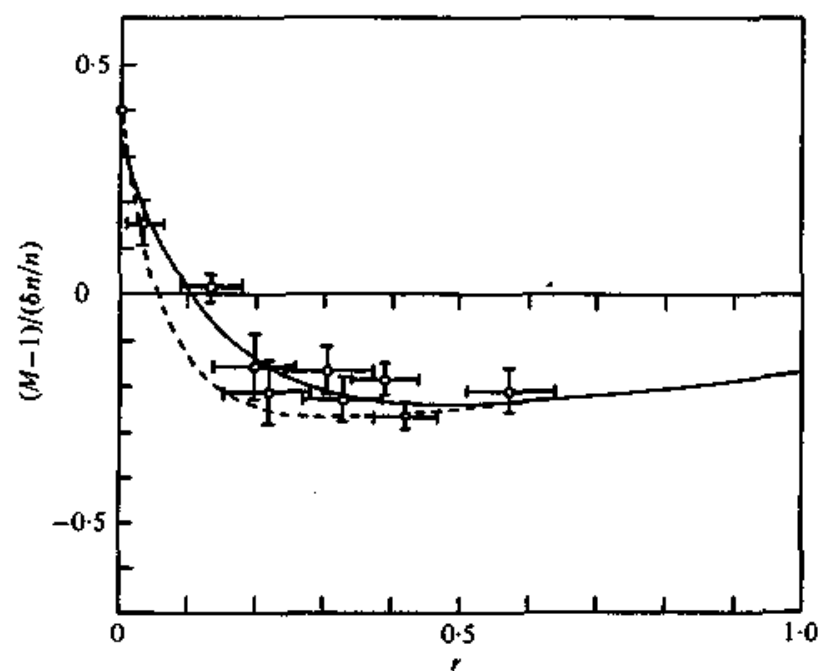

FigURE 6. Measured $(M-1)$ divided by the normalized height or depth as a function of the negative ion concentration $r$. The solid and dotted curves are obtained from (3) when $T_{1} / T_{\mathrm{e}}=0$ and $0 \cdot 1$, respectively.

dotted curves are $A / 3 B S$ calculated from (3) when $T=0$ and $0 \cdot 1$, respectively. The non-zero ion temperature makes the ion-acoustic wave less dispersive with the results that the speed of solitons becomes faster for the same amplitude than when $T=0$. The open eircles in the upper plane correspond to compressive solitons except the one for $r=0 \cdot 13$. The point for $r=0.13$ corresponds to the positive pulse shown in figure 5 . The data in the lower half plane are for rarefactive solitons, that is, their density perturbations $\delta n / n$ are negative. The experimental results are considered to agree with the theoretical prediction.

Another characteristic which is often compared with the theory for the $\mathrm{KdV}$ soliton is the dependence of the width $D$ of solitons on the amplitude. Measurements indicate that the product of the square of the width and the amplitude is constant within experimental errors for a fixed $r$. The width is defined as the full width at the height of 0.786 times the amplitude of solitons. Measured products $\left(D / \lambda_{e}\right)^{2}(\delta n / n)$ versus the negative ion concentration are summarized in figure 7 whose upper and lower half planes correspond to compressive and rarefactive solitons, respectively. The open circle on the vertical axis is the case when $r=0$. Equation (4) is also shown by the solid and dotted curves when $T=0$ and $0 \cdot 1$, respectively. The wave becomes less dispersive owing to the non-zero ion temperature, which makes the width of rarefactive solitons narrower than when $T=0$. It is similar to compressive solitons $(r=0)$ studied by Watanabe (1975). The result shown in figure 7 is considered to agree with the prediction of the KdV equation.

The properties of the solitary negative waves described above seem to indicate that the waves are solitons. However, if the observed solitary waves are solitons, they have to keep their identity after collisions (Drazin 1980, p. 8). To confirm 


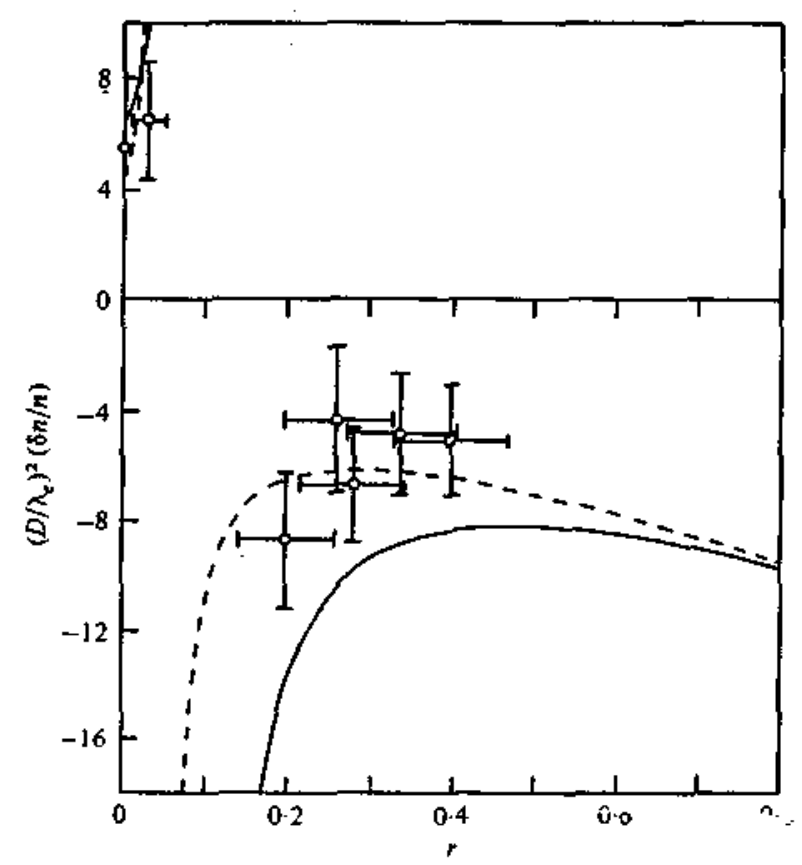

FraURE 7. Square of the normalized width times the normalized height or depth of solitons versus the negative ion concentration. The solid and dotted curves show (4) for $T_{1} / T_{0}=0$ and $0 \cdot 1$, respectively.

the present solitary negative waves to be solitons, collision experiments have been performed, which will be described below.

First, overtaking collisions are observed by applying two negative sinusoidal pulses which excite two successive solitons at the source anode. When the amplitude of the leading soliton is much smaller than the overtaking one, the second catches up with the first. On the other hand, when both amplitudes are comparable (figure 8), the larger soliton becomes smaller as it approaches the leading soliton. At the same time, the amplitude of the leading soliton increases, so that the overtaking soliton cannot catch up with the other. These phenomena are the same as those observed for compressive solitons in a plasma without negative ions (Ikezi, Taylor \& Baker 1970).

Next, head-on collisions of rarefactive solitons were investigated in a tripleplasma device (Nakamura, Nomura \& Itoh 1977). The device is constructed with addition of another source to the double-plasma device. The target chamber is replaced by a shorter one of $24 \mathrm{~cm}$ in length. The solitons propagating in opposite directions are transmitted by applying a negative sinusoidal pulse to both anodes of the right and left source chambers. An axially movable cylindrical probe instead of the plane probe is used to detect counter-propagating solitons. An example of observed head-on collisions is shown in figure $\mathbf{9}$, which is similar to those observed for compressive solitons (Ikezi, Taylor \& Baker 1970; Nakamura 1982). The solitons pass through without change of their shape. The results of the overtaking and head-on collisions described above confirm that the present negative solitary waves are rarefactive solitons. 


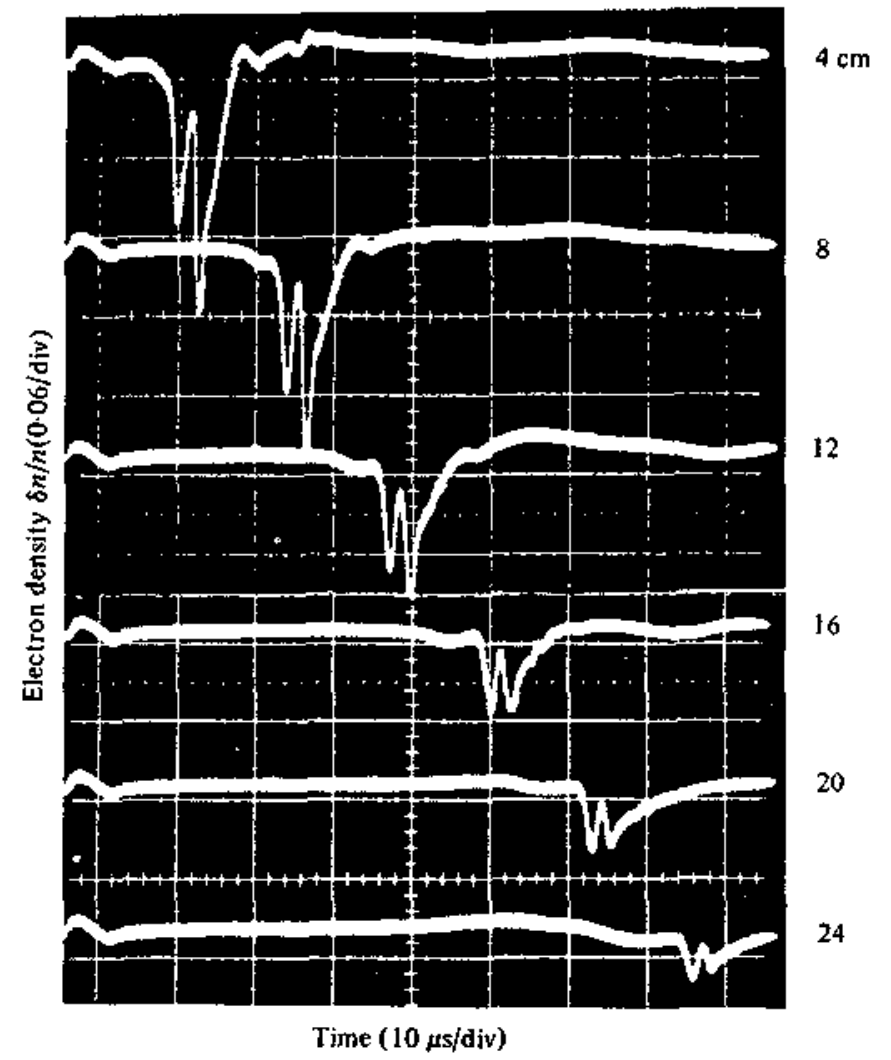

FIGURE 8. Observed signals for an overtaking collision of rarefactive solitons of comparable amplitudes. $r \simeq 0.23$. The figures indicate the distance from the grid.

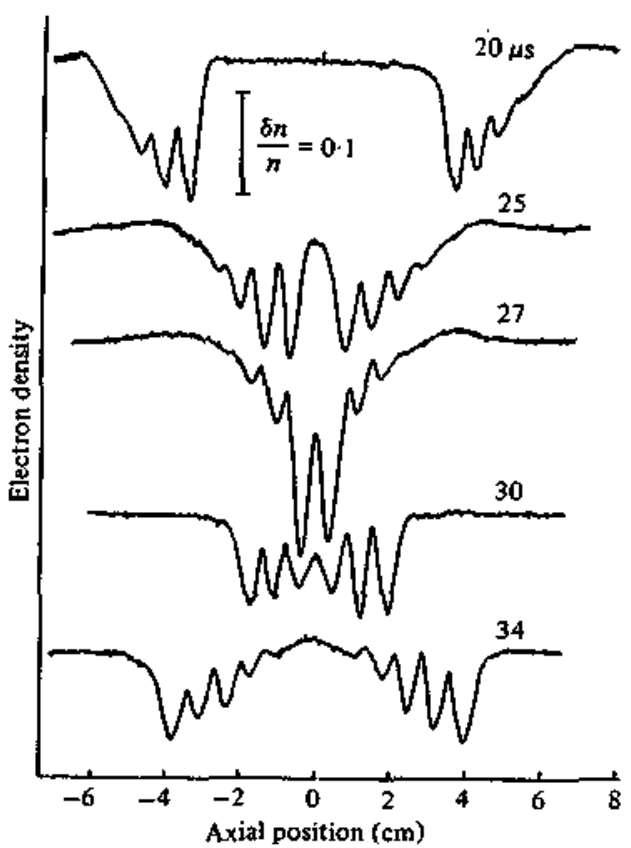

Fradre 9. A collision of rarefactive solitons propagating in opposite directions. $r \simeq 0.48$. The figures show the time that has elapsed since the pulse was applied to the anodes. 


\section{Conclusions}

Rarefactive solitons which have been predicted to exist in a multi-component plasma with negative ions are observed experimentally when the concentration of the negative ions is larger than a certain critical value. The measured characteristics of the rarefactive solitons agree with the Korteweg-de Vries equation derived from a fluid model of the plasna, which takes the ion temperature into account. However, better agreements are expected by taking multicomponents of both positive and negative ions and higher-order nonlinearity neglected in the Korteweg-de Vries equation into consideration.

In compressive, positive solitons in a plasma without negative ions, there are trapped electrons (Pierre el al. 1983) and resonant ions known as a precursor (Karpman et al. 1980). On the other hand, in the present rarefactive, negative solitons, trapped ions are thought to exist, whose effect might be different from the trapped electrons in the compression solitons, owing to their much heavier mass. The trapped ions are not investigated in the present experiment, but will be the subject of a future paper.

One of the authors (Y.N.) would like to thank Professors N. Yajima, Y. Itikawa and $\mathrm{S}$. Watanabe for discussions and encouragement.

\section{REFERENCES}

Asundi, R. K. \& Craggs, J. D. 1964 Proc. Phys. Soc. 83, 611.

D'Angero, N., Gohler, S. V. \& OHE, T. 1966 Phys. Fluids, 9, 1605.

DAS, G. C. 1977 Plasma Phys. 19, 363.

DAS, G. C. 1979 Plasma Phys, 21, 257.

Das, G. C. \& Tagare, S. G. 1975 Plasma Phys. 17, 1025.

Drazrn, P. G. 1983 Solitons. Cambridge University Press.

ICHIKAWA, Y. H. \& WATANABE, S. 1977 J. de Phys. C6-15, 38.

IKEZr, H. 1973 Phys. Fluids, 16, 1663.

IkezI, H. 1978 Solitons in Action (ed. K. E. Lonngren and A. Scott), p. 153. Academic.

IKEZX, H., TAYLOR, R. J. \& BAKBR, D. R. 1970 Phys, Rev. Lett. 25, 11.

Karpuan, V.I., Lywov, J.P., Michelsen, P., Pzostitu, H. L., Rasmussen, J.J. \& Trrkov, V. A, 1980 Phys. Fluids, 23, 1782.

Lonngren, K. E. 1983 Plasma Phys. 25, 943.

LUdwg, G. O., Ferretra, J. L. \& Nakamura, Y. 1984 Phys. Rev. Lett. 52, 275.

NAKAMURA, Y. 1982 IEEE Trans. Plasma Sci. PS-10, 180.

NaKamURA, Y., NakamURA, M. \& ITOH, T. 1976 Phys. Rev. Lett. 37, 209.

NakamuRa, Y., Nomura, Y. \& IтоH, T. 1977 Phys. Rev. Lett. 39, 1622.

Naramura, Y. \& NomURA, Y. 1980 Phys. Lett. $75 \mathrm{~A}, 351$.

Nakamura, Y. \& TsukabaYashi, I. 1984 Phys. Rev. Lett. 52, 2356.

Okdtsd, E. \& Nalamura, Y. 1979 Plasma Phys. 21, 1053.

Pierae, T., Bonhomise, G., Gussenot, J. R. \& Leclert, G. 1983 J. Physigue, 44, 629.

Tran, M. Q. 1979 Physica Scripta, 20, 317.

Tran, M. Q. \& Coquhrand, S. 1976 Phys. Rev. A14, 2301.

Watanabe, S. $1975 \mathrm{~J}$. Plasma Phys. $14,353$.

Wong, A. Y., Mamas, D. L. \& ARNUSH, D. 1975 Phys. Fluids, 18, 1489. 


\begin{tabular}{|c|c|}
\hline $\begin{array}{c}\text { PROPOSTA PARA } \\
\text { PUSLICACAOO }\end{array}$ & $\begin{array}{l}\square \text { DISSERTAC̄AOO } \\
\square \text { TESE } \\
\otimes \text { RELATÓRIO }\end{array}$ \\
\hline & Б OUTROS \\
\hline
\end{tabular}

EXPERIMENTS ON ION-ACOUSTIC RAREFACTIVE SOLITONS IN A MULTI-COMPONENT PLASMA WITH NEGATIVE IONS
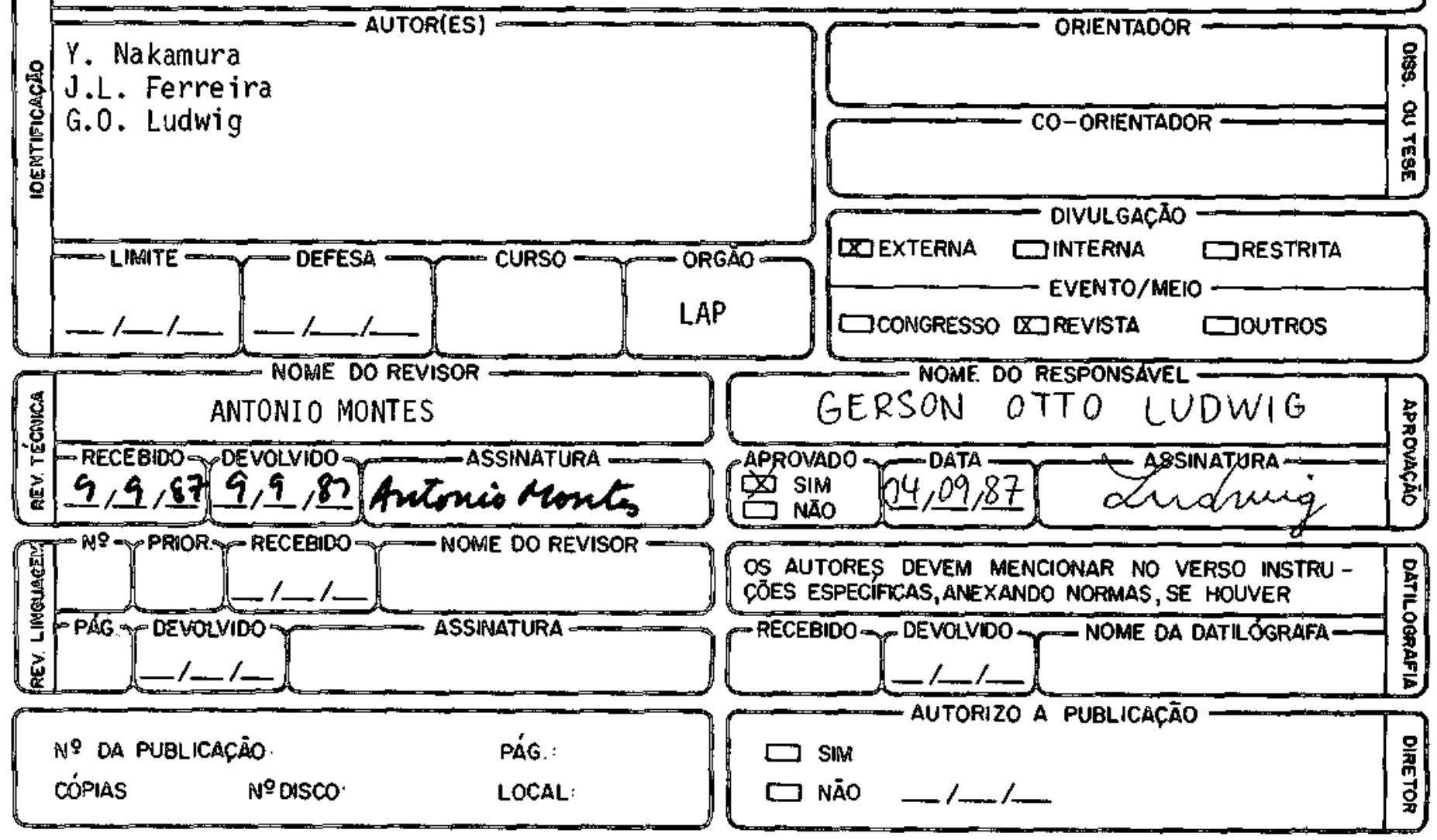

OESERVAÇŌES E NOTAS

Poasificacat: PRE 